\title{
ANALYSIS OF HIGH - STRENGTH STEEL PIN CONNECTION
}

\author{
Josef VIČAN ${ }^{1, *}$, Matúš FARBÁK ${ }^{1}$ \\ ${ }^{1}$ Department of Structures and Bridges, Faculty of Civil Engineering, University of Žilina, Univerzitná \\ 8215/1, 01026 Žilina, Slovakia. \\ corresponding author: vican@uniza.sk.
}

\begin{abstract}
The paper presents analytical and numerical analysis of the pin connection resistance made of high-strength steel used in the temporary bridge ŽM 60. The assessment of the pin connection was performed according to standard [1] and also using numerical analysis by means of numerical sophisticated calculations based on FEM analyses.
\end{abstract}

\section{Keywords:}

High-strength steel; Pin connection; Lock,

Truss bridge;

Numerical analysis.

\section{Introduction}

In the frame of cooperation with praxis, Department of structures and bridges was involved into working up the analysis of pin connection resistance connecting the lower chords of the main truss girders of the temporary bridge ŽM 60. The superstructure of the bridge ŽM 60 represents a demountable structure used for quick bridging of small and medium obstacles. It is applicable on the railways up to a span of $60 \mathrm{~m}$. Generally, it can also be used on roads, but for larger spans, which is usually not usable, because its maximum use of up to $60 \mathrm{~m}$ is respected also on the roads.

\section{Description of bridge ŽM 60 superstructure}

The demountable railway bridge ŽM 60 [2] is a steel truss structure designed for the quick erection of bridges of medium spans. It consists of precisely manufactured components connected by screw connections and pins. Due to the theoretical height of $7.00 \mathrm{~m}$, the bridge is closely arranged by means of the upper bridge bracing. The lower plated bridge deck is discreetly connected by crossbeams to the main girders of every $3.00 \mathrm{~m}$. The axial distance of the main girders of $5.40 \mathrm{~m}$ allows the use of a free width of the bridge of $5.00 \mathrm{~m}$ and when it is used as a road bridge with steel safety barriers connected to the main girders, the free width of the bridge is $4.80 \mathrm{~m}$ only.

Main bridge girders having span up to $60.00 \mathrm{~m}$ are statically designed as rigid chords stiffened by the trusses of the oblique systems without verticals. Their lower chords are created as welded Ishaped beams. The web made of P $8 \times 760 \mathrm{~mm}$ is widened into a Y-shape inserting angles $140 \times 140$ $\times 8 \mathrm{~mm}$ in contact with the flanges, which support wide flanges made of $P 16 \times 400 \mathrm{~mm}$, provided at both ends with forged ridge locks for longitudinal connection of parts of $6.00 \mathrm{~m}$ long by seven-shear pin connections.

The end lower chord is stiffened on the one side by end verticals and end plates for use of a pull-out arm formed also by an end front-connected chord. The upper chord is made of an $\mathrm{H}$-shaped welded steel profile and its web made of $P 10 \times 350 \mathrm{~mm}$ is lightened by thirteen circular holes with a diameter of $240 \mathrm{~mm}$. Flanges made of P $25 \times 350 \mathrm{~mm}$ are provided at each end with twelve holes with a diameter of $31 \mathrm{~mm}$ for connecting the gussets of the bridge upper bracing members. In the middle part, there are four holes for connecting the diagonals of the bridge upper bracing. Diagonals of truss girders are made of welded $\mathrm{H}$ profiles with flanges made of $\mathrm{P} 16 \times 300 \mathrm{~mm}$ and webs made of $\mathrm{P} 10 \times$ $368 \mathrm{~mm}$. At both ends, the flanges are provided with six holes having a diameter of $31 \mathrm{~mm}$ for connection to the gussets of the lower and upper chords. The web is lightened by seventeen holes with a diameter of $240 \mathrm{~mm}$. All elements of the main girders of the ŽM 60 bridge are made of steel 15 422.5 . 
The lower plated bridge deck consists of individual segments $3.00 \mathrm{~m}$ long with a width of 4.958 $\mathrm{m}$, which are connected to the lower chords of the main girders. The bridge deck segment consists of the $8 \mathrm{~mm}$ thick steel sheet stiffened in the transverse direction by two l-shaped crossbeams and in the longitudinal direction by 8 trapezoidal stiffeners and 4 longitudinal flat stiffeners made of IPE 220 rolled profiles. The crossbeams are provided at the ends with end-plates with 8 holes having a diameter of $31 \mathrm{~mm}$ for connecting the bridge segment to the lower chords of the main girders. The surface of the bridge deck is covered with a layer of plastic concrete replacing the carriage when using the structure as a temporary road bridge. All bridge elements are made of steel S355J0 and S355J2 [3].

The bridge is installed on standard fixed and movable bearings $360 \mathrm{~mm}$ high, directly mounted on the bridge recesses of abutments. The bearings are the parts of the bridge ŽM 60 superstructure and they are made of 16224.6 steel [4]. Bridge expansion joints are made of bridge deck end plates, which are the parts of the ŽM 60 bridge set.

The actual pin connection of the main girders is made of steel bars of $\phi 50 \mathrm{~mm}$. The pin is designed from special steel 03Cr10Ni10MO2 [5] with following strength characteristics: $f_{y}=1500$ $\mathrm{MPa}, f_{u}=1600 \mathrm{MPa}$. The description of the pin connection is documented by photos in Fig. 1 and drawing in Fig. 2.
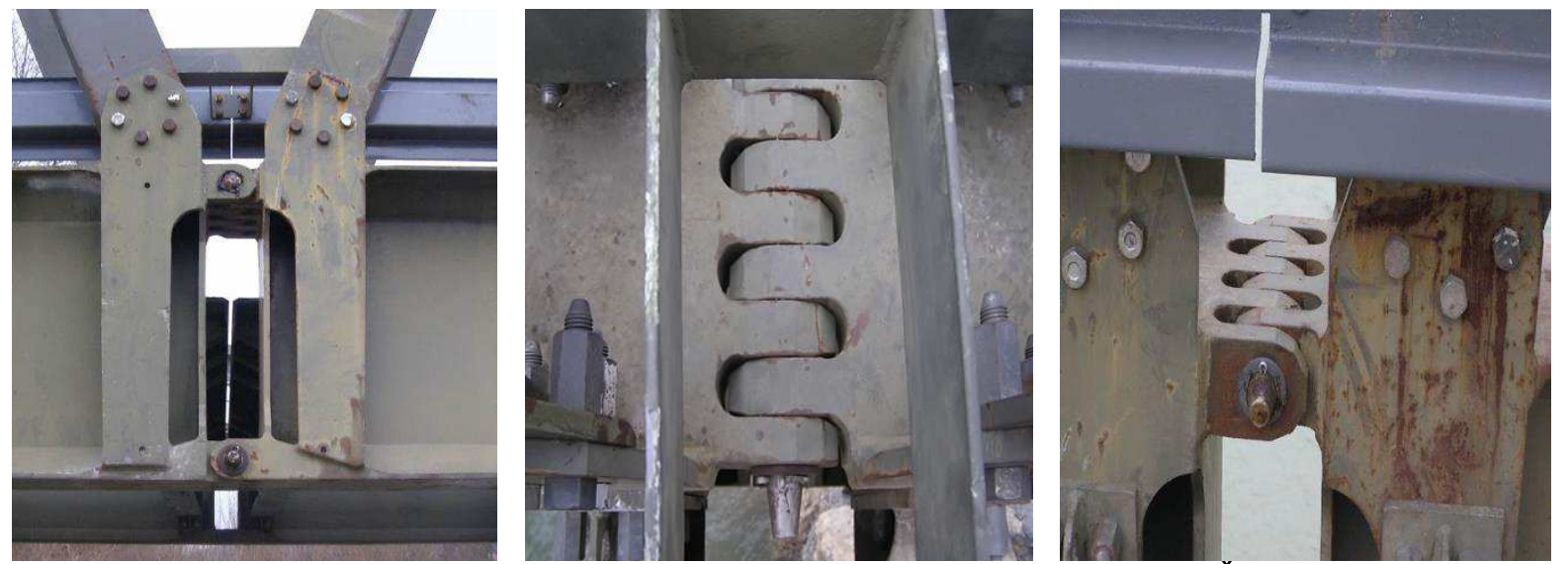

Fig. 1: Pin connection (lock) of the lower chord of the bridge ŽM 60.

\section{Analysis of pin connection}

\subsection{Assessment of pin connection according to STN EN 1993-1-8}

\subsubsection{Verification of pin connection geometrical parameters}

The geometry of a pin connection should fulfil rules according to standard [1], which are presented in Table 1. Comparing the geometrical parameters of pin connection from Fig. 2 to requirements in Table 1, it has been found that the pin connection of the ŽM 60 bridge chords does not meet the stated requirements. If the given thickness of connecting parts $t=52 \mathrm{~mm}$ is respected, the required length $a$ should be according to tab. 1 in the value of $a=57.6 \mathrm{~mm}$, while the real dimension is only $a=44.5 \mathrm{~mm}$.

Table 1: Geometrical requirements for pin ended members.

\begin{tabular}{|l|l|}
\hline \multicolumn{2}{|c|}{ TYPE A: Given thickness } \\
\hline
\end{tabular}



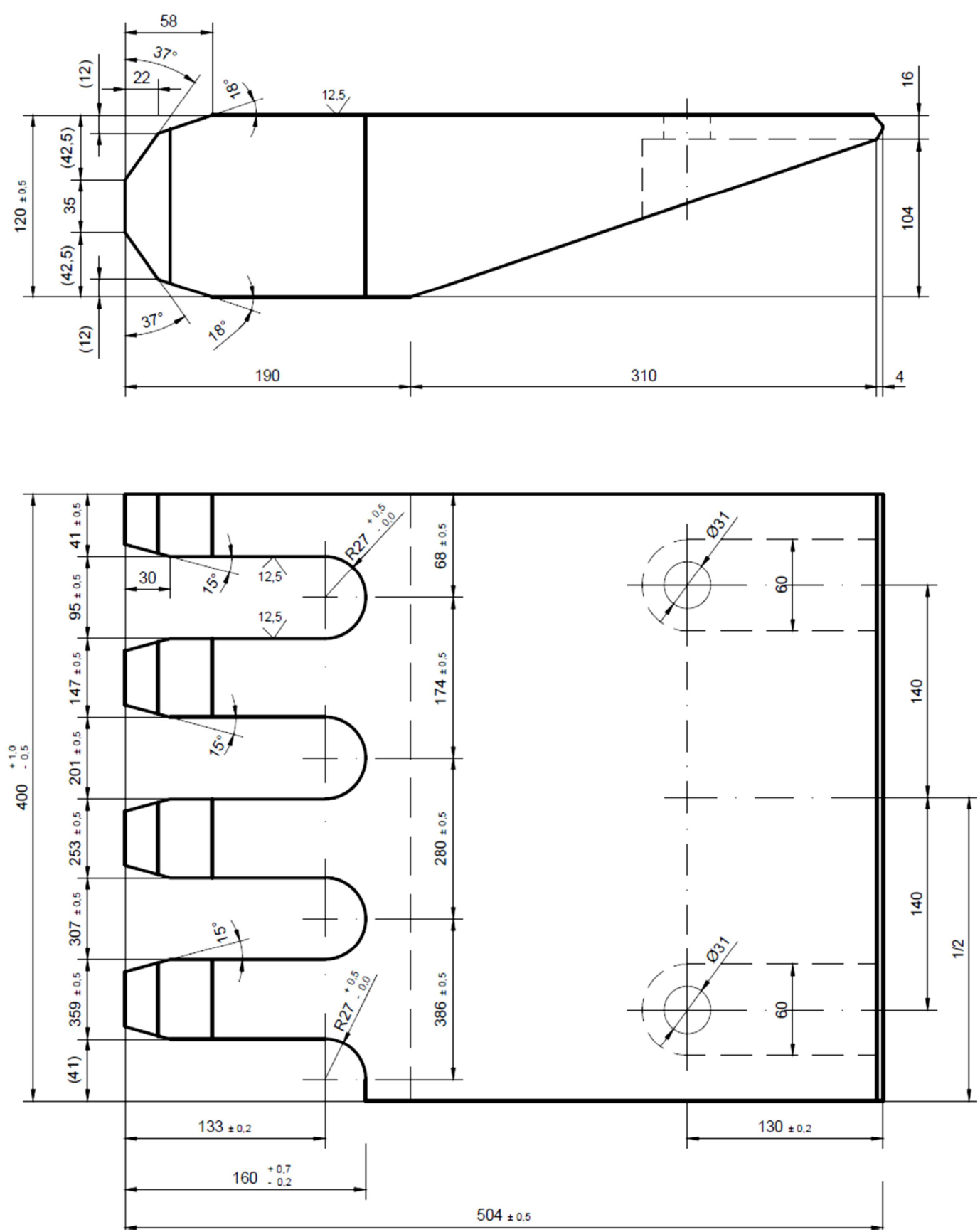

Fig. 2: Drawing of the connecting parts (lock).

Also, dimension of the parameter $c$ should be $c=44.6 \mathrm{~mm}$, but its real value reaches $c=34.5$ $\mathrm{mm}$ only. In the recommended formulas, the force $F_{E d}$ was determined as the tensile resistance of the bridge lower chord.

\subsubsection{Verification of pin connection resistance}

Pin connections of the lower chords of the ŽM 60 bridge shall satisfy the design requirements according to standard [1] given in Table 2. 
Table 2: Design criteria for pin connections.

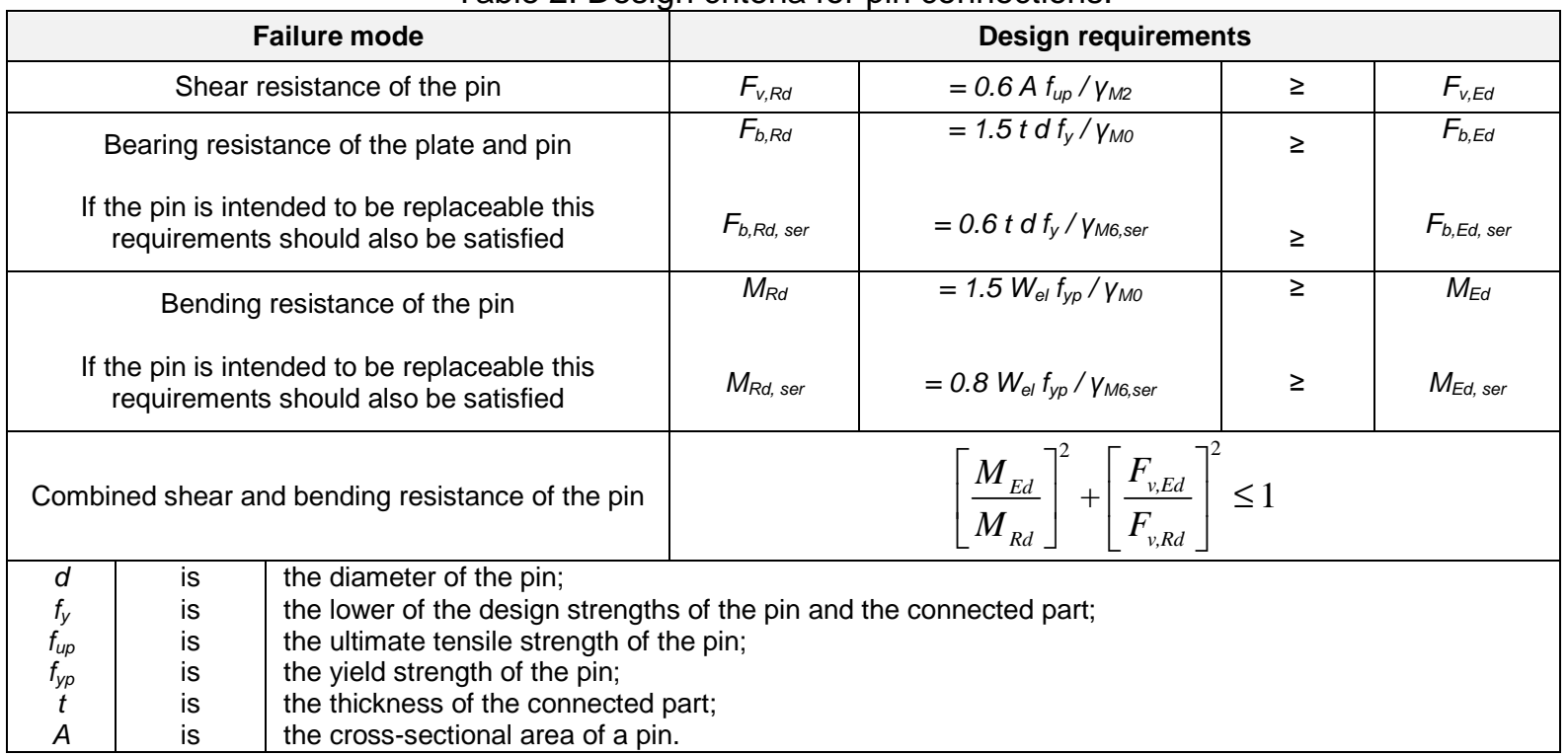

The force $F_{E d}$ was determined as the tensile resistance of the lower chord and its value was $F_{E d}$ $=6291.06 \mathrm{kN}$. The value shall be transmitted by the observed pin connection in the case if it is not to be a limiting element of the bridge superstructure. In that manner, determined force $F_{E d}$ loading the lock was calculated for one tooth of the lock in the value of $F_{v, E d}=1660.58 \mathrm{kN}$. The shear resistance belonging to one tooth of the lock was $F_{V, R D}=1507.96 \mathrm{kN}$, from which it is clear that the pin transfers only $90.8 \%$ of the lower chord resistance. In addition to shear, the pin is also stressed by a bending moment $M_{E d}=33.21 \mathrm{kNm}$, but its bending resistance determined according to the relation from Table 2 is equal to $M_{R d}=27.612 \mathrm{kNm}$. Thus, in terms of the pin bending resistance, the pin transfers only $83.1 \%$ of the lower chord resistance. But from the viewpoint of the combination of shear and bending loading, the pin resistance is at the level of $63.1 \%$ of the lower chord resistance. Since according to standard procedures the pin is not satisfactory, the more accurate analysis of the pin resistance by numerical analysis using FEM was necessary to perform.

\subsection{Numerical analysis of pin connection resistance}

The numerical model of the pin connection was created in the software environment based on the finite element method [6]. The geometric parameters of the model correspond as much as possible to the actual shape and dimensions of the pin connection. The material of the connected parts - lock, as well as the pin itself, was considered to be elastic-plastic, bi-linear with strain hardening with the following parameters:

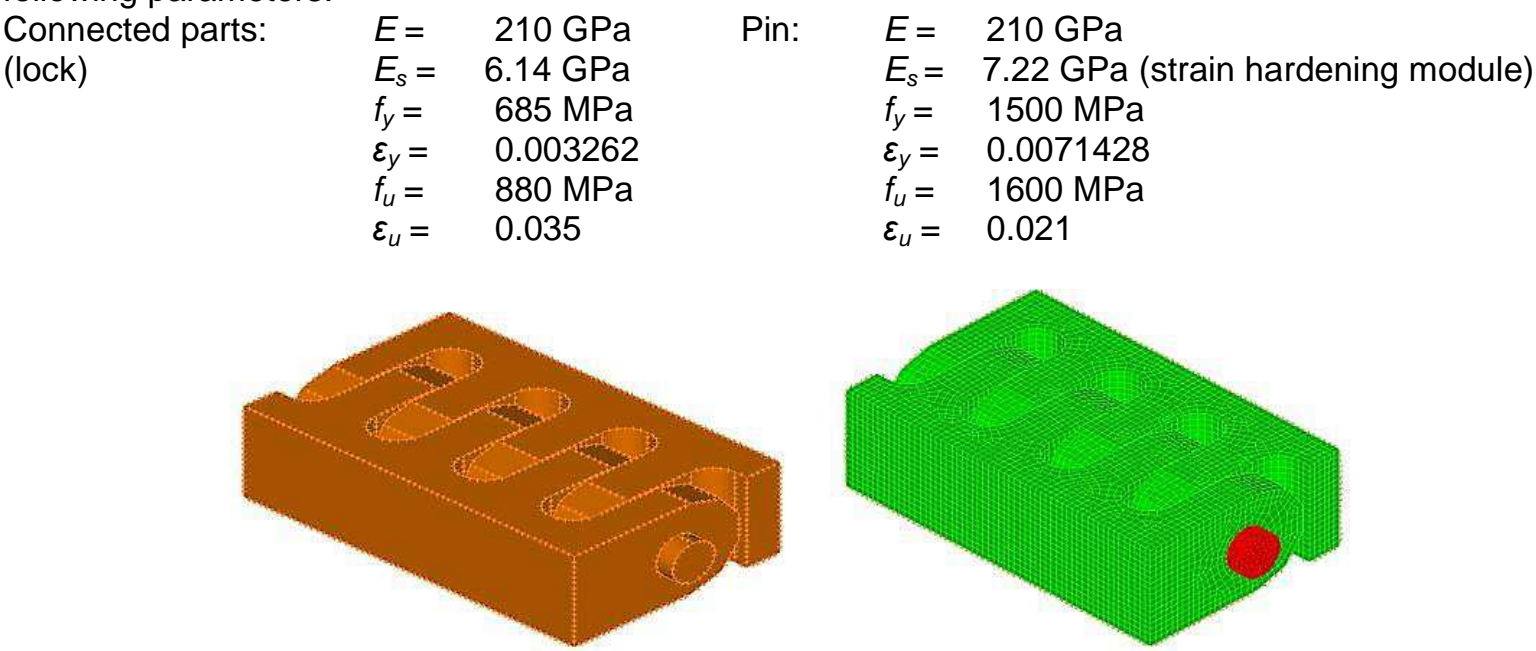

Fig. 3: Pin connection geometry and finite elements meshing. 
The contact between the connected parts and the pin body was solved as a 3D contact with the definition of individual surfaces of both bodies and their harmonization into contact pairs [7]. The value of Coulomb friction between the pin and the lock was considered to be 0.1 [8]. A finite element network of approximately $7 \mathrm{~mm}$ in size was created by an automatic generator using solid eight-node finite elements, preferring their regular block shape.

The boundary conditions were chosen to best simulate the position and behaviour of the connection in the whole structure [9]. The load of the connection by the force $F_{E d}=6291.06 \mathrm{kN}$ (given by the tensile resistance of the lower chord [10]) is considered as a uniform load acting on a surface with dimensions of $120 \times 400 \mathrm{~mm}$. The connection was loaded with a gradual force increase in 20 loading steps. The nonlinear analysis was performed using the Newton-Raphson iteration method, using the energy convergence criterion [11].

The results of the numerical analysis showed that the plasticization of the connection occurs at the full value of the load. The deformation of the connection is documented in Fig. 4.
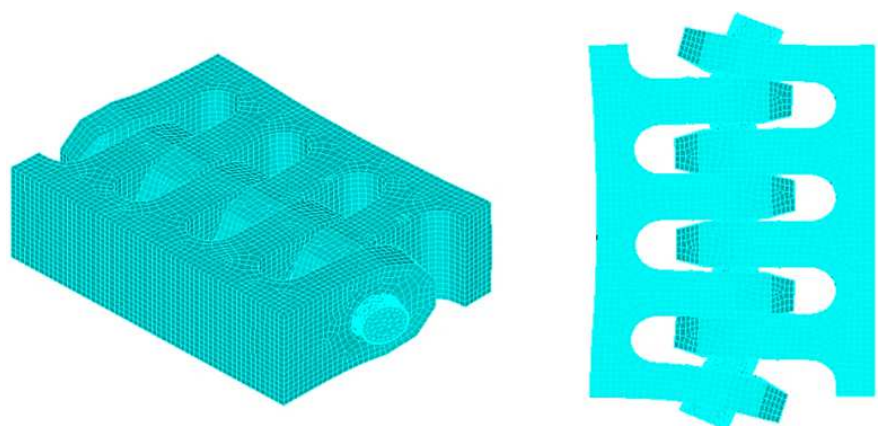

Fig. 4: Deformation of the lock and the pin.

The stresses in the pin reached up to $1600 \mathrm{MPa}$ and they are further redistributed. The course of the stresses on the surface of the pin is presented in Fig. $5 \mathrm{a}$, the stress redistribution along the pin cross-section is documented in Fig. $5 \mathrm{~b}$. When the connection was subjected to the force $F_{E d}=6$ $291.06 \mathrm{kN}$, the total deformation of the pin (relative displacement of points 1 and 2) represented a value of $1.2 \mathrm{~mm}$ (see Fig. 6).



a)



b)

Fig. 5: Stresses on the pin surface (a) and pin cross-section (b).

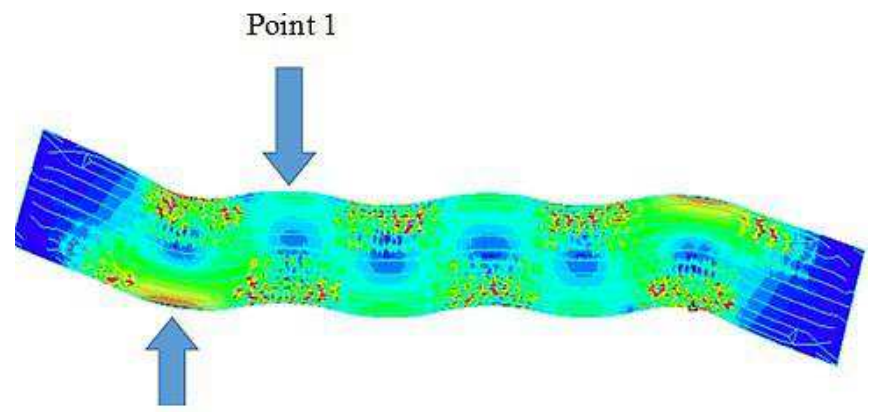

Point 2

Fig. 6: Pin deformation. 
In addition to the stresses of the pin, the stresses of the connected parts (lock) were also observed, as it is made of lower quality steel than the pin. The stress course in the individual teeth of the lock is presented in Fig. 7. The stress was determined at the force $F_{E d}=4656.00 \mathrm{kN}$. This force can be considered from the viewpoint of the ultimate limit state to be decisive for the correct function of the lock and the pin. It is the force at which the first plasticization of the lock teeth occurs.

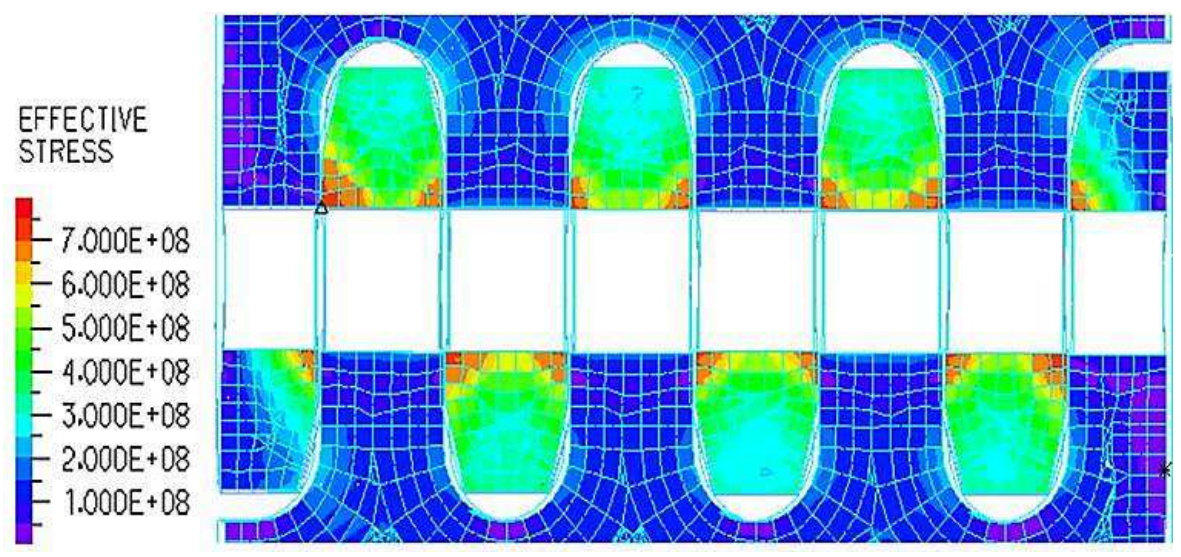

Fig. 7 Stress course in the lock.

\section{Conclusion}

Based on the analytical calculation of the pin resistance according to the standard [1], it was found that its resistance corresponds to $61.3 \%$ of the lower chord resistance of the truss main girder of the bridge ŽM 60 . Numerical analysis of the pin connection also proved the need to verify the lock (connected parts), as it is made of lower steel quality (steel 15422.5 ) and therefore it is decisive from the viewpoint of the ultimate limit state of the chord pin connection, as the geometry of the pin connection does not meet the requirements of the standard [1]. If reaching the yield strength at the most stressed point of the cross-section of the lock and the pin is assumed as the limit state, then the material of the lock decides. The limit force that the lock transmits is then $4656.00 \mathrm{kN}$, which represents $74.0 \%$ of the lower chord resistance of the main truss girder.

\section{Acknowledgements}

The paper presents the results of the research project No. 1/0336/18 supported by the Slovak Grant Agency.

\section{References}

[1] STN EN 1993-1-8: Design of steel structures - Part 1-8: Design of joints. SUTN Bratislava 2005.

[2] REGULATION ŽM 60. Basic information about bridge ŽM 60.

[3] STN EN 1993-1-1: Design of steel structures. Part: 1-1: General rules and rules for buildings. SUTN Bratislava 2006.

[4] BENDIS, A.: The Fatigue Properties of 16 224. 6 Steel and Its Welded Joints. Zvaranie, 1985, 34.10, pp. 308-312.

[5] Analysis of the bridge ŽM 60. Vítkovice, 1982. Proceeded by Ing. Novotný.

[6] VIČAN, J. - FARBÁK, M.: Report from analysis of the pin connection of the bridge ŽM 60 lower chord. Department of Structures and Bridges, UNIZA 02/2019.

[7] ADINA, R. D. INC.: ADINA Theory and Modeling Guide, ADINA R\&D, Watertown, Massachusetts, USA 2012.

[8] ANH LE XUAN: Dynamics of mechanical system with coulomb Friction. Foundation of Engineering Mechanics, Springer 2003, Berlin

[9] KREJSA, M. et al: Numerical analysis of fatigue damage on selected connection of the crane bridge support structure. In: Engineering mechanics 2018: book of full texts. Prague: The Czech Academy of Science. The Institute of Theoretical and Applied Mechanics, 2018, pp. 437-440.

[10] VIČAN, J. at all: Existing Steel Railway Bridges Evaluation. In: Civil and Environmental Engineering. Vol. 12, Issue 2, 2016, pp. 103-110.

[11] DE BORST, R. - CRISFIELD, M. A. - REMMERS, J. J. - VERHOOSEL, C. V.: Nonlinear finite element analysis of solids and structures. John Wiley \& Sons, 2012. 